\title{
If I Feel Ticklish, I Will Keep My Distance, but If I Feel Itchy, I Will Approach You: Ticklish and Itchy Sensations Influence the Interpersonal Distance
}

\author{
Yasuto Okamura $^{1,2} \&$ Mitsuhiro Ura ${ }^{3}$ \\ ${ }^{1}$ Graduate School of Psychology, Otemon Gakuin University, Osaka, Japan \\ ${ }^{2}$ Japan Society for the Promotion of Science, Tokyo, Japan \\ ${ }^{3}$ Department of Psychology, Otemon Gakuin University, Osaka, Japan \\ Correspondence: Yasuto Okamura, Gradute School of Psychology, Otemon Gakuin University, Osaka, Japan. \\ E-mail: yasu0night.person@gmail.com
}

Received: December 7, 2018

Accepted: December 19, 2018

Online Published: December 27, 2018

doi:10.5539/ijps.v11n1p1

URL: https://doi.org/10.5539/ijps.v11n1p1

\begin{abstract}
Studies have found metaphor-consistent effects for certain cutaneous sensations. However, no research to date has investigated the influence of ticklish and itchy sensations on social proximity. This study examined possible metaphor-consistent effects between these sensations and social proximity. Participants looked at a photograph inducing a ticklish or an itchy sensation and responded to a questionnaire inquiring about interpersonal distances. Results indicated that participants in the tickling condition desired to maintain more distance to medium-intimate others, whereas those in the itchy condition desired to maintain less distance. These findings confirmed the metaphor-consistent effects of these sensations on social proximity. Theoretical implications of this finding are discussed.
\end{abstract}

Keywords: metaphor, cutaneous sensations, social proximity

\section{Introduction}

Lakoff and Johnson (1980, p.5) explained that "the essence of metaphor is understanding and experiencing one kind of thing in terms of another", and proposed the conceptual metaphor theory (CMT) in which metaphors create meanings at a cognitive level. CMT consists of two elements: a source and a target concept. Lakoff and Johnson (1980) pointed out that metaphors function as conceptual mappings between source concepts and superficially dissimilar target concepts. One characteristic of these concepts is that source concepts represent concrete knowledge and embodied experiences. Target concepts, in contrast, represent more abstract knowledge that is difficult to comprehend. Psychological research has demonstrated that manipulating source or target concepts result in the activation of the other concept in a metaphor consistent manner (see Landau, Meier, \& Keefer, 2010). This phenomenon is known as the "metaphor consistent effect". Williams and Bargh (2008), for example, found that touching a warm cup increases impressions of warmth and friendliness toward another person "AFFECTION IS WARMTH". Jostmann, Lakens, and Schubert (2009) reported that holding heavy things enhances the cognition of importance "IMPORTANCE IS HEAVY". Meier and Robinson (2004) showed that objects presented higher in a visual space are estimated more positively, whereas those presented lower are estimated negatively "AFFECTION IS VERTICAL SPACE". These examples support the metaphor consistent effect.

Although many findings have accumulated on metaphor consistent effects, no studies have elucidated the effects of ticklish and itchy sensations, which are assumed to be similar. For example, Kumar (2002) indicated that ticklish and itchy sensations are both simultaneously occurring subliminal forms of cutaneous "pain". However, even though these sensations have a commonality, they have different metaphoric expressions in linguistic usage. In Japanese, kayui (itchy) insinuates a sense of frustration and impatience and kusuguttai (ticklish) insinuates a sense of embarrassment. Kövecses (2005) suggested that actual linguistic metaphors are expressions of conceptual metaphors. Therefore, it is reasonable to assume that different metaphor consistent effects would occur for ticklish and itchy sensations. 
The distinction between ticklish and itchy sensations could be explained by their origin. The itchy sensation has the function for getting rid of noxious insects as can be observed in the grooming behavior of animals. However, grooming occurs not only for keeping the body surface clean but also for increasing the sense of friendship and security among in-group members (Morris, 1968). Therefore, it is possible that the itchy sensation could enhance sociability and approach reactions directed at others. On the other hand, the ticklish sensation is more complicated than the itchy sensation, and its physiological basis and mechanism remain obscure. Suzuki and Yamashita (2016) suggested that the ticklish sensation produces ambivalent reactions, such as laughing (a positive dimension) and avoidance behaviors (a negative dimension). However, Hall and Allin (1897) and Harris (2012) indicated that the ticklish sensation could be divided into two dimensions: gargalesis (the sensation that induces laughing) and knismesis (the sensation caused by a light touch that does not induce laughing). We focused exclusively on knismesis and highlighted only the negative dimension of the ticklish sensation.

The identical distinction between the two sensations can be made from a physiological perspective. The itchy sensation has been defined as "an unpleasant cutaneous sensation which provokes the desire to scratch" (Rothman, 1941, p.357), which implies that the itchy sensation and the desire to scratch are indivisible. The ticklish sensation, on the other hand, is associated with withdrawal and defensive movements (Harris \& Alvarado, 2005). In fact, being tickled is assumed to automatically generating bodily movements for brushing something off the skin. Consequently, the itchy sensation might be related to approach reactions for realizing a desire, whereas the ticklish sensation might be linked to avoidance reactions. Therefore, both metaphoric linguistic expressions and physiological findings support the distinction between these two sensations.

This study examined whether ticklish and itchy sensations affected approach-avoidance behaviors in a metaphor-consistent manner. We speculated that these sensations would have the function of adjusting interpersonal distances because approach-avoidance reactions influence interpersonal distances. Therefore, we estimated the conceptual metaphor "SOCIAL PROXIMITY IS AN ITCHY/TICKLISH SENSATION" and hypothesized that metaphor consistent effects would occur in the conceptual metaphor, which is related to "SOCIAL PROXIMITY IS WARMTH" (IJzerman \& Semin, 2009; Landau, Zhong, \& Swanson, 2018). Studies supporting this connection have found that warmer temperatures lead individuals to perceive others as being socially closer and more intimate, whereas cold temperatures lead to perceptions of distance and rejection. Regarding the relationship between the itchy sensation and the perception of warmth, it is known that an itch is frequently induced by a warm environment especially in patients with dermatoses (Murota et al., 2012). Moreover, the relationship between the ticklish sensation and the perception of coldness could be that both cause a withdrawal response (Chew, Oon, Lee, Lim, \& Tan, 2010). Therefore, the metaphor "SOCIAL PROXIMITY IS AN ITCHY/TICKLISH SENSATION" could be possible.

To test this hypothesis, we estimated interpersonal distances using the scene imagination method. Also, we assessed the extraversion of participants based on the finding of previous classical studies showing that extraverts maintained a shorter distance during conversations and had smaller personal spaces (Cook, 1970; Williams, 1963). Based on these findings, we analyzed the influence of the two sensations on interpersonal distances by using the degree of participants' extraversion as a covariate to avoid the influence of the covariate on interpersonal distances.

\section{Method}

\subsection{Ethics Statement}

The study was approved by the Academic Committee of the Department of Social Psychology at Otemon Gakuin University. Participants were informed that they could drop out at any time during the survey. Their informed consent was obtained before they took part in the study.

\subsection{Participants}

Sample sizes were calculated using G*Power 3.1.9.2 (Faul, Erdfelder, Lang, \& Buchner, 2007) and the parameters alpha $=0.05$, the power $=0.8$, and the effect size $\mathrm{f}=.25$ based on Cohen (1988). Participants were undergraduate students $(N=174,68$ men and 106 women; aged 18 to 30 years), who received partial course credits participating in the study. They were given the questionnaires and responded to them during a psychology class.

\subsection{Stimuli and Design}

Colored pictures of green foxtail grass (Setaria viridis) and a T-shirt tag (Appendix) were used as the independent variables and responses to a questionnaire inquiring about the participants' interpersonal distance was used as the dependent variable. It was assumed that the picture of green foxtail grass would lead to a ticklish 
sensation, whereas the picture of the tag would lead to an itchy sensation. A between-participants one-way analysis of variance (ANOVA) was used to analyze differences between the conditions.

\subsection{Procedure}

Participants were randomly assigned to the tickling $(N=63)$ or the itchy $(N=63)$, or the control condition $(N=$ 48). All participants first responded to the 16-item extraversion scale that was based on Goldberg (1992) and Saucier (1997), which included items such as, I am the life of the party. All items were rated on a 5-point Likert scale ranging between 1 (strongly disagree) and 5 (strongly agree). After that, the participants conducted one of the two experimental conditions for 30 seconds: either (1) looking at the picture of green foxtail grass, or (2) looking at the tag while imagining that they were touching the nape of their neck. Then, they responded to the questionnaire inquiring about their interpersonal distance by using the scene imagination method. The following scene was described to the participants to facilitate their imagination: "When you are walking in the university, you see a same sexed person whose face and name are known to you, but you have not talked to before. The person did not see you." In the scene imagination method, we treated the relationships between the target person and participants as having a mid-level of intimacy based on Sasaki, Sugawara, and Tanno (2005). The participants were asked to describe how they would behave in this situation by using a scale ranging from 1 (you totally ignore the person) to 9 (you are willing to talk to the person). Participants were also asked to describe how they would feel by using nine items: shy, clumsy, miserable, timidly, bashful, embarrassed, nervous, irritatied, and tantalizing. Seven items (shy, clumsy, miserable, timidly, bashful, embarrassed, and nervous) were adopted as "Shyness", based on Sugawara (1998) and Higuchi (2000). The two items (irritatied and tantalizing) were adopted as "Irritation" and have metaphorical meanings in Japanese that are related to the itchy sensation. The presentation order of the nine items was randomized by presenting them in a block-wise manner. Finally, as a manipulation check the participants were asked to indicate how strongly ticklish or itchy they would feel if the grass or the tag would touch the nape of their neck by using a scale ranging from 1 (not at all) to 5 (extremely). Participants in the control condition responded to the 16-item extraversion scale and the scene imagination survey. They responded the questionnaire in a separate room from those in the experimental conditions considering the different duration of the task.

\section{Results}

We first conducted an ANOVA to confirm whether participants felt ticklish or itchy when looking at each of the two pictures, which indicated significant differences between the two pictures. This demonstrated that the participants felt more ticklish for the grass $(M=3.78, S E=.18)$ than for the tag $(M=3.21, S E=.18)(F(1,124)$ $=5.33, p=.023, \eta_{\mathrm{p}}{ }^{2}=.04,95 \% \mathrm{CI}=.00$ to .13$)$, whereas they felt more itchy for the tag $(M=3.79, S E=.16)$ than for the grass $(M=2.56, S E=.16)\left(F(1,124)=28.80, p<.001, \eta_{\mathrm{p}}{ }^{2}=.19,95 \% \mathrm{CI}=.08\right.$ to .31$)$. A one sample t-test indicated that these means were significantly higher than 3.0 , which was the theoretical median of the scales measuring ticklish and itchy sensations $(t(62)=4.84, p<.001,95 \% \mathrm{CI}=.46$ to 1.10 for the ticklish sensation and $t(62)=5.16, p<.001,95 \% \mathrm{CI}=.49$ to 1.10 for the itchy sensation). Then, we performed an analysis of covariance (ANCOVA) with interpersonal distance as the dependent variable and conditions (tickling, itchy, and control) as the independent variables, with the sum of the scores of the 16 items as a covariate. This indicated significant effects of the covariate $\left(F(1,170)=34.20, p<.001, \eta_{\mathrm{p}}{ }^{2}=.17,95 \% \mathrm{CI}=.08\right.$ to .26$)$ and conditions $\left(F(2,170)=28.10, p<.001, \eta_{\mathrm{p}}{ }^{2}=.25,95 \% \mathrm{CI}=.14\right.$ to .34$)$. We also conducted a multiple comparison tests on the effect of the conditions, which indicated that all conditions showed significant differences. The tickling condition $(M=2.57, S E=.22)$ showed less personal distance than the itchy condition $(M=4.87, S E=.22, p<.001)$ or the control condition $(M=3.39, S E=.25, p=.013)$, whereas the itchy condition showed more personal distance than the control condition $(p<.001)$. We also conducted an ANOVA without the covariate, which showed results consistent with the ANCOVA and indicated significant differences between the three conditions $\left(F(2,171)=20.04, p<.001, \eta_{\mathrm{p}}{ }^{2}=.19,95 \% \mathrm{CI}=.09\right.$ to .29$)$. Multiple comparison tests revealed that the tickling condition $(M=2.67, S E=.24)$ showed less distance than the itchy condition $(M=4.75$, $S E=.24, p<.001)$ or the control condition $(M=3.40, S E=.27, p=.043)$, whereas the itchy condition showed more distance than the control condition $(p<.001)$.

A statistical mediation analysis (MacKinnon, 2008) was conducted to test for mediation effects of participants' degree of shyness and irritation on the relationship between the condition and interpersonal distance. The results of the mediation analysis with the degree of participants' feelings as the mediator indicated that their interpersonal distance was not mediated by their feelings, because neither the relationship between independent variables and shyness $(\beta=.07, t=.80, p=.43)$ nor irritation $(\beta=.07, t=.74, p=.46)$ was significant. 


\section{Discussion}

The results supported the hypothesis that the ticklish sensation enhances avoidance reactions and the itchy sensation increases the approach reaction directed at others, suggesting that the data could be explained by the conceptual metaphor "SOCIAL PROXIMITY IS AN ITCHY/TICKLISH SENSATION". The origins of itchy and ticklish sensations discussed in the introduction suggest that the itchy sensation is accompanied by a desire for scratching, which is an approach movement towards the stimulus to get rid of an object on the skin surface. On the contrary, the ticklish sensation, especially knismesis, is associated with the avoidance reaction of slapping something away. This study is the first to examine metaphor consistent effects of ticklish and itchy sensations on interpersonal distance and demonstrated that activating the source concepts of itchy or tickling sensations activates the target concept (social proximity) in a metaphor-consistent manner. This finding underlines the importance of the distinction between the itchy and the tickling sensation. Certainly, these sensations have the commonality that they occur when something is lightly touching the surface of the skin. We speculated that individuals could discern between the itchy and the tickling (knismesis) sensation.

The mediational effects of the participants' moods on the metaphor consistent process were not confirmed in the study, suggesting that the sensations directly influenced the interpersonal distance regardless of feelings, which are also an important factor affecting social proximity. This direct influence is consistent with previous findings. For example, Jostmann, Lakens, and Schubert (2009) examining the metaphor "IMPORTANCE IS HEAVY" reported that holding a heavy clipboard increased judgments of monetary value. The effect of the clipboard weight on evaluations of the value of a currency remained significant even when the mood was controlled by an ANCOVA. Considering those results, the lack of a mediational effect of the mood could be attributed to the direct associations between the source and the target concepts.

In conclusion, the results of this study indicated that itchy and tickling sensations moderated interpersonal distance and confirmed that there is a conceptual metaphor "SOCIAL PROXIMITY IS AN ITCHY/TICKLISH SENSATION".

\section{Future Directions}

This study has certain limitations that need to be addressed in the future. Firstly, it would be necessary to focus on cultural differences of metaphor consistent effects in future studies. In fact, itchy and ticklish are metaphorically different in English and Japanese. In English, itchy insinuates a strong desire for something (e.g., I'm just itching to get to know all of you better!) and ticklish insinuates much joy or pleasure (e.g., I was tickled pink to have successfully made myself understood in English.). Lee and Schwarz (2012) pointed out that metaphors may have a universal structure (e.g., smell-suspicion, documented in at least 18 languages) with culture-specific content (e.g., fishy in English, unspecified in Chinese and German). Based on this assumption, we speculate that conceptual metaphors of itchy and ticklish sensations have both cross-cultural and culture-specific dimensions. If so, these sensations might influence Japanese and English speakers differently. Another limitation is that we did not itch or tickle participants but used photographs that induced these sensations. Certain studies have shown that embodied experience and imagination of the experience has the same effects, and that imagination is a virtual bodily movement (Tsukimoto, 2001). For example, Porro et al. (1996) found that the same regions of the brain are activated in both actual and imaginary movements. Moreover, Tsukimoto (2005) suggested that imagination is a neurological activity without muscular movements that excites the peripheral nervous system. Based on this knowledge, itching or tickling participants and showing stimuli that give rise to those sensations might have similar effects on interpersonal distance, although the effect size might be different. Solving these limitations of the study will provide additional support to research on metaphor.

\section{Acknowledgments}

This study was supported by JSPS KAKENHI (Grant Number 18J15436).

\section{References}

Chew, K. S., Onn, L. L., Lee, R. A., Lim, K. S., \& Tan, C. T. (2010). Withdrawal response in healthy adults. Neurology Asia, 15(2), 159-165. Retrieved from https://www.neurology-asia.org/

Cohen, J. (1988). Statistical power analysis for the behavioral sciences $2^{\text {nd }}$ Edition. Hillsdale, NJ: Lawrence Erlbaum.

Cook, M. (1970). Experiments on orientation and proxemics. Human Relations, 23, 61-76. https://doi.org/10.1177/001872677002300107 
Faul, F., Erdfelder, E., Lang, A.G., \& Buchner, A. (2007). G*Power 3: a flexible statistical power analysis program for the social, behavioral, and biomedical sciences. Behavior Research Methods, 39, 175-191. https://doi.org/10.3758/BF03193146

Goldberg, L. R. (1992). The development of markers for the Big-Five factor structure. Psychological Assessment, 4, 26-42. https://doi.org/10.1037/1040-3590.4.1.26

Hall, G. S., \& Allin, A. (1897). The psychology of tickling, laughing, and the comic. American Journal of Psychology, 9, 1-44. https://doi.org/10.2307/1411471

Harris, C. R. (2012). Tickling. In V. Ramachandran (Eds.), Encyclopedia of Human Behavior $2^{\text {nd }}$ Edition, 611-615. Amsterdam: Elsevier. https://doi.org/10.1016/B978-0-12-375000-6.00364-5

Harris, C. R., \& Alvarado, N. (2005). Facial expressions, smile types, and self-report during humour, tickle, and pain. Cognition \& Emotion, 19, 655-669. https://doi.org/10.1080/02699930441000472

IJzerman, H., \& Semin, G. R. (2009). The thermometer of social relations: Mapping social proximity on temperature. Psychological Science, 20, 1214 - 1220. https://doi.org/10.1111/j.1467-9280.2009.02434.x

Jostmann, N. B., Lakens, D., \& Schubert, T. W. (2009). Weight as an embodiment of importance. Psychological Science, 20, 1169-1174. https://doi.org/10.1111/j.1467-9280.2009.02426.x

Kövecses, Z. (2005). Metaphor in culture: Universality and variation. Cambridge, England: Cambridge University Press. https://doi.org/10.1017/CBO9780511614408

Kumar, S. L. (2002). Homoeopathy in Rheumatism. India: B. Jain Publishers.

Lakoff, G., \& Johnson, M. (1980). Metaphors We Live By. Chicago, IL: University of Chicago Press.

Landau, M. J., Meier, B. P., \& Keefer, L. A. (2010). A metaphor-enriched social cognition. Psychological Bulletin, 136, 1045-1067. https://doi.org/10.1037/a0020970

Landau, M. J., Zhong, C. B., \& Swanson, T. J. (2018). Conceptual metaphors shape consumer psychology. Consumer Psychology Review, 1, 54-71. https://doi.org/10.1002/arcp.1002

Lee, S. W. S., \& Schwarz, N. (2012). Bidirectionality, mediation, and moderation of metaphorical effects: the embodiment of social suspicion and fishy smells. Journal of Personality and Social Psychology, 103, 737-749. https://doi.org/10.1037/a0029708

MacKinnon, D. P. (2008). Introduction to statistical mediation analysis. New York, NY: Lawrence Erlbaum.

Meier, B. P., \& Robinson, M. D. (2004). Why the sunny side is up. Psychological Science, 15, 243-247. https://doi.org/10.1111/j.0956-7976.2004.00659.x

Morris, D. (1968). The naked ape: A zoologist's study of the human animal. London: Jonathan Cape.

Murota H, Izumi M, Abd El-Latif M. I., Nishioka, M., Terao, M., Tani, M., Matsui, S., Sano, S., \& Katayama, I. (2012). Artemin causes hypersensitivity to warm sensation, mimicking warmth-provoked pruritus in atopic dermatitis. Journal of Allergy and Clinical Immunology, 130(3), 671-682. https://doi.org/10.1016/j.jaci.2012.05.027

Porro, C.A., Francescato, M.P., Cettolo, V., Diamond, M.E., Baraldi, P., Zuiani, C., Bazzochi, M., \& di Prampero, P.E. (1996). Primary motor and sensory cortex activation during motor performance and motor imagery: A functional magnetic resonance study. The Journal of Neuroscience, 16, 7688-7698. http://doi.org/10.1523/JNEUROSCI.16-23-07688

Rothman, S. (1941). Physiology of itching. Physiological Reviews, 21, 357-381. https://doi.org/10.1152/physrev.1941.21.2.357

Sasaki, J., Sugawara, K., \& Tanno, Y. (2005). Why is the diverse U letter relationship between embarrassment and psychological distance observed?: In view of Schlenker and Leary (1982) 's self-presentation model of social anxiety. The Japanese Journal of Psychology, 76(5), 445-452. https://doi.org/10.4992/jjpsy.76.445

Saucier, G. (1997). Effects of variable selection on the factor structure of person descriptors. Journal of Personality and Social Psychology, 73, 1296-1312. https://doi.org/10.1037//0022-3514.73.6.1296

Suzuki, M., \& Yamashita, H. (2016). Effects of predictability of tactile stimulus on perception of the tickle. Bulletin of the Faculty of Education, Ehime University, 63, 109-112. Retrieved from http://www.ed.ehime-u.ac.jp/ kiyou/ 
Tsukimoto, H. (2001). Embodied AI: Symbol grounding through imagination. AAAI Fall Symposium on Anchoring Symbols to Sensor Data in Single and Multiple Robot Systems, 67-74. Retrieved from https://aaai.org/Symposia/Fall/fss18.php

Tsukimoto, H. (2005). Embodied semantics. Journal of the Japan Association for Philosophy of Science, 33, 31-40. https://doi.org/10.4288/kisoron1954.33.31

Williams, J. L. (1963). Personal space and its relation to extraversion-introversion. Canadian Journal of Behavioural Science/Revue canadienne des sciences $d u$ comportement, 3(2), 156-160. https://doi.org/10.1037/h0082257

Williams, L. E., \& Bargh, J. A. (2008). Experiencing physical warmth influences interpersonal warmth. Science, 322, 606-607. https://doi.org/10.1126/science.1162548

\section{Appendix}

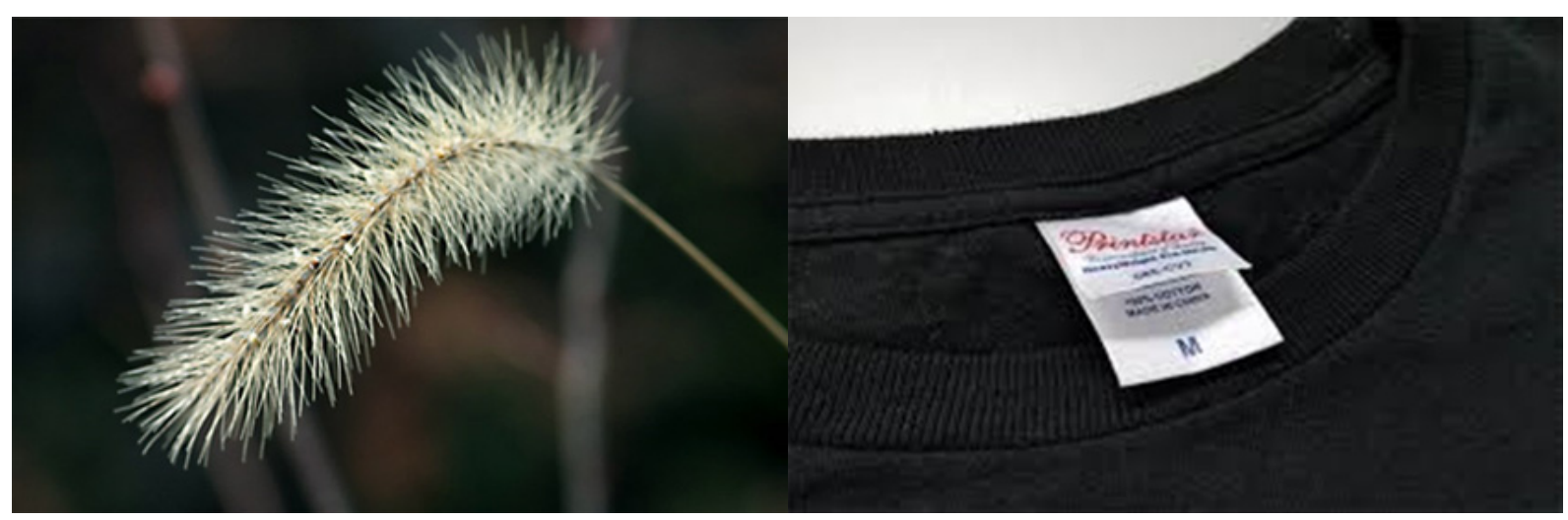

\section{Copyrights}

Copyright for this article is retained by the author(s), with first publication rights granted to the journal.

This is an open-access article distributed under the terms and conditions of the Creative Commons Attribution license (http://creativecommons.org/licenses/by/4.0/). 